EXTENDED REPORT

\title{
Standard reference values for musculoskeletal ultrasonography
}

\author{
W A Schmidt, H Schmidt, B Schicke, E Gromnica-Ihle
}

Ann Rheum Dis 2004;63:988-994. doi: 10.1136/ard.2003.015081

See end of article for authors' affiliations

\section{Correspondence to:} Dr W A Schmidt, Medical Centre for Rheumatology Berlin-Buch, Karower Strasse 11, 13125 Berlin; schmidt.wa@t-online.de

Accepted 29 December 2003

\begin{abstract}
Objectives: To determine standard reference values for musculoskeletal ultrasonography in healthy adults. Methods: Ultrasonography was performed on 204 shoulders, elbows, hands, hips, knees, and feet of 102 healthy volunteers (mean age 38.4 years; range 20-60; 54 women) with a linear probe (10-5 MHz; Esaote Technos MP). Diameters of tendons, bursae, cartilage, erosions, hypoechoic rims around tendons and at joints were measured with regard to established standard scans. Mean, minimum, and maximum values, as well as two standard deviations (2 SD) were determined. Mean values \pm 2 SD were defined as standard reference values.

Results: Hypoechoic rims were normally present in joints and tendon sheaths owing to physiological synovial fluid and/or cartilage. Similarly, fluid was found in the subdeltoid bursa in 173/204 (85\%), at the long biceps tendon in $56(27 \%)$, in the suprapatellar recess in $158(77 \%)$, in the popliteal bursae in 32 $(16 \%)$, and in the retrocalcaneal bursa in $49(24 \%)$. Erosions of $>1 \mathrm{~mm}$ were seen at the humeral head in $47(23 \%)$. Values for important intervals were determined. The correlation between two investigators was 0.96 (0.78-0.99). The reliability of follow up investigations was $0.83(0.52-0.99)$.

Conclusions: Fluid in bursae as well as hypoechoic rims within joints and around tendons are common findings in healthy people. This study defines standard reference values for musculoskeletal ultrasonography to prevent misinterpretation of normal fluid as an anatomical abnormality.
\end{abstract}

M usculoskeletal ultrasonography is a diagnostic method which has been in use for decades. Improvements in technology have led to its increasingly widespread use in rheumatology, ${ }^{2-6}$ orthopaedic surgery, ${ }^{78}$ sports medicine, ${ }^{9}{ }^{10}$ physical therapy, ${ }^{11}$ and radiology. ${ }^{12}$ One of its main indications is the need to distinguish between normal and pathological anatomical structures if clinical findings are ambivalent. Nevertheless, standard reference values have not yet been established for this important diagnostic method. Technological advances have enhanced the resolution of ultrasound images, leading to the ability to detect synovial fluid and cartilage in healthy subjects. Definition of standard reference values is therefore necessary to increase the diagnostic accuracy of ultrasonography.

\section{SUBJECTS AND METHODS Test subjects}

We performed bilateral investigation of 102 white volunteers recruited from among our staff, students, and friends of the authors. We excluded subjects aged $<20$ or $>60$ years, or with a history of inflammatory rheumatic disease, osteoarthritis, or surgery of any of the investigated joints. None of the subjects developed an inflammatory rheumatic disease 9-11 months after measurement. Table 1 shows the demographic data of subjects.

\section{Ultrasonography}

An experienced physician sonographer (HS) performed the investigations and measurements based on standard scans. ${ }^{13}$ We chose the anatomical structures for measurement that we considered most relevant for daily practice. Tables $2-5$ show the anatomical structures, the ultrasound planes, and the exact localisation of measurement. Figure 1 delineates the measurement of the finger flexor tendons. Figure 2 shows the measurement at the ankle joint. We saved all images on the hard disk of the ultrasound equipment. In 20 subjects, another trained physician sonographer (WAS) repeated measurement of the saved images. In two people the same investigator repeated the complete investigation after 1 and 9 months: for one person by HS, and for one by WAS.

We employed a linear probe for all investigations (LA 523, 10-5 MHz; length of the probe, $45 \mathrm{~mm}$; weight of probe 100 g; year of construction 2002; Esaote Technos MP; Esaote SpA; Genua, Italy). Scanner settings were uniform for all measurements: frequency setting, $10 \mathrm{MHz}$; B mode gain $110 \%$; one focus point position in the region of measurement; procession parameter, PB 5; scan correlation parameter, SCC 7; and enhancing parameter, ENH 5. Bilateral measurement for one test subject took 2 hours. The axial spatial resolution for this probe is $0.154 \mathrm{~mm}$; the lateral spatial resolution is $0.260 \mathrm{~mm}$. The pressure on the probe was below the occurrence of visible deformation of the anatomical structures.

We additionally measured all distances in two subjects to compare the data with technology which had less resolution: ATL (Philips) HDI 3500 (12-5 MHz, length of probe, $38 \mathrm{~mm}$; year of construction 1998; Advanced Technology Laboratories, Bothell, WA, USA). Both the axial and the lateral spatial resolution of this probe are $0.7 \mathrm{~mm}$.

For the investigation of the shoulders the sonographer sat behind the subject. The subject sat with $90^{\circ}$ flexion of the elbow. We investigated the biceps tendon in neutral position; the subscapularis tendon in maximum external rotation; and the supraspinatus and infraspinatus tendons, the acromioclavicular and sternoclavicular joint, the subdeltoid bursa, and the acromiohumeral distance in $60^{\circ}$ internal rotation. We measured the largest erosion detected at the humeral head. We defined an "erosion" as a pit in the bone surface of $>1 \mathrm{~mm}$ diameter in all three diameters (longitudinal, transverse, sagittal).

Abbreviations: MCP, metacarpophalangeal; MRI, magnetic resonance imaging; PIP, proximal interphalangeal 
Table 1 Demographic data of the subjects investigated in this study

\begin{tabular}{llll}
\hline & Women & Men & All subjects \\
\hline Number & 54 & 48 & 102 \\
Age (years) & 37.8 & 39.0 & 38.4 \\
Right handed & 50 & 44 & 94 \\
Mean body weight (kg), & $62(38-94)$ & $80(62-103)$ & $70(38-103)$ \\
range & & & \\
Mean height (cm), range & $166(156-$ & $179(167-$ & $172(156-$ \\
Good working activity (n) & 18 & $198)$ & $198)$ \\
\hline
\end{tabular}

Occupations: physician (13); salesperson (14); administrative assistant (7); skilled worker (6); student or engineer (5 each); lawyer or teacher (4 each); manager, nurse, physiotherapist, car mechanic, or construction worker ( 3 each); photographer, advertising assistant, kindergarten teacher, policeman or woman (2 each); secretary, psychologist, speech therapist, bank employee, television producer, programmer, doctor's assistant, pharmacist, dentist, beautician, stewardess, florist, roofer, cook, painter, varnisher, tiler, machine operator, cleaner, truck driver (1 each).

The investigator sat in front of the subject for investigation of the elbows. The subject sat with full extension of the elbow and supination of the lower arm for the anterior scans, and with maximum flexion for the posterior scan.

The investigator sat in front of the subject for investigation of hands and fingers. The subject sat with the hand on top of the thigh, with supination for the volar scans, and with pronation for the dorsal scans in neutral position of the wrist.

For investigation of hips, knees, and feet, the subject was placed prone or supine, with the investigator sitting next to him or her.

For hips, the subject was placed supine with the hip joint in neutral position.

Knees were investigated with the subject placed supine for the anterior scans and prone for the posterior scans, with the knee joint in neutral position. We measured only the intercondylar cartilage with maximum flexion of the knee. Subjects contracted the quadriceps muscle for evaluation of the suprapatellar and lateral recess, the patellar tendon, and the deep infrapatellar bursa.

The examiner investigated feet with the subject placed supine for the anterior scans and prone for the posterior and plantar scans. Anterior scans took place with $90^{\circ}$ flexion of the knee, with the sole of the foot resting on the examination

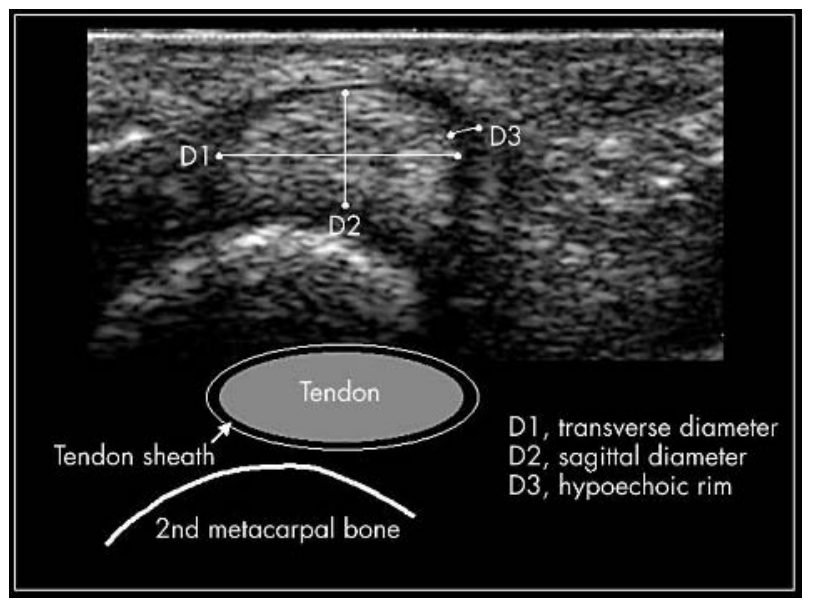

Figure 1 Ultrasound image with measurement of the flexor tendons of the right index finger $1 \mathrm{~cm}$ proximal to the metacarpophalangeal (MCP) joint (transverse volar scan). The hypoechoic rim of all tendons was measured at the point of the largest diameter in the images.

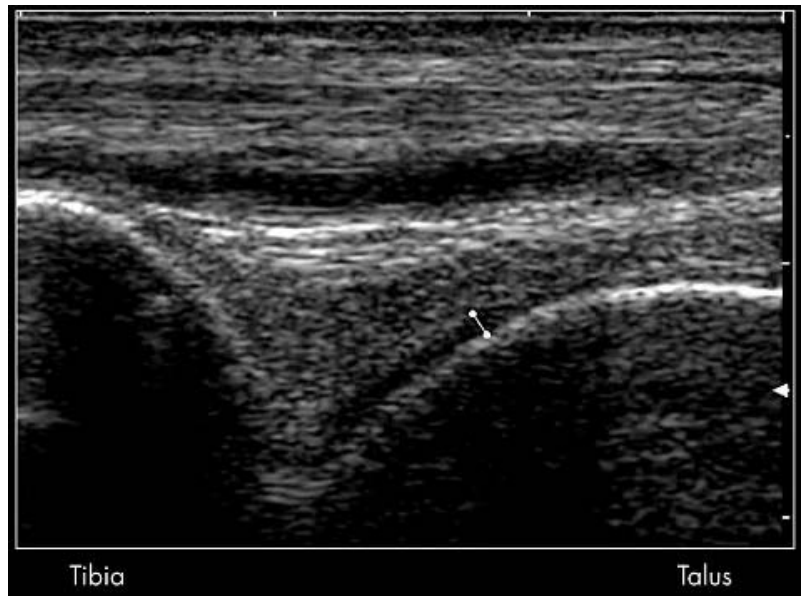

Figure 2 Ultrasound image with measurement of the anterior bonecapsule distance of the tibiotalar joint (anterior longitudinal scan).

table. We performed posterior and plantar scans with the foot relaxed and hanging off the edge of the examination table.

We measured all bone-capsule distances perpendicular to the bone.

\section{Statistical analysis}

We saved data in a structured query language database and employed SPSS (version 11.0) for processing. Our work produced mean, minimum, and maximum values, as well as two standard deviations (2 SD). We used an analysis of variance to compare the mean values. We evaluated qualitative features by contingency table analysis and the non-parametric $\chi^{2}$ test. We correlated measured values with age, sex, body weight, and height, sport activities, and hand dominance. We used the paired $t$ test for correlated samples to compare the observers.

\section{RESULTS}

Tables 2-5 show mean, minimum, and maximum values, as well as 2 SD of the measurements. We have defined standard reference values as those values included in $2 \mathrm{SD}$.

\section{Shoulders}

We detected fluid around 56/204 (27\%) long biceps tendons. The diameter of the supraspinatus tendon was larger than those of the subscapularis and the infraspinatus tendons. We found erosions of $>1 \mathrm{~mm}$ at the humeral head in 47 (23\%) shoulders. We determined fluid in $173(85 \%)$ subdeltoid bursae. For the capsular distension of the axillary recess, 2 SD includes diameters $\leqslant 3.8 \mathrm{~mm}$. The mean transverse diameter of the long biceps tendon was $4.6 \mathrm{~mm}(2.1-7.2 ; 2 \mathrm{SD}, 2.0)$ in women and $5.5 \mathrm{~mm}(3.3-8.8 ; 2 \mathrm{SD}, 2.0)$ in men. The mean sagittal diameter was $2.5 \mathrm{~mm}$ (1.5-6.0; $2 \mathrm{SD}, 1.4)$ in women, and $2.8 \mathrm{~mm}(1.7-5.2 ; 2 \mathrm{SD}, 1.4)$ in men. In 11 acromioclavicular joints of nine patients we detected small osteophytes that indicate preclinical osteoarthritis.

\section{Elbows}

We made images of capsular distension in all coronoid and olecranon fossae. The anterior ulnar distance between bone and capsule primarily represents cartilage thickness.

\section{Hands, wrists, and fingers}

The 2 SD include distances $\leqslant 2.9 \mathrm{~mm}$ and $\leqslant 3.4 \mathrm{~mm}$ for the dorsal and volar capsular distension at the scaphoid bone, respectively; $\leqslant 1.6 \mathrm{~mm}$ and $\leqslant 2.1 \mathrm{~mm}$ at the dorsal and volar aspect of the ulna, respectively; and $\leqslant 1.9 \mathrm{~mm}$ and $\leqslant 1.6 \mathrm{~mm}$ 
Table 2 Anatomical structure with ultrasound plane; localisation of measurement; mean, maximum, and minimum value; and two standard deviations at the shoulders and the sternoclavicular joints

\begin{tabular}{|c|c|c|c|c|c|c|}
\hline Anatomical structure & Ultrasound plane & Exact localisation & $\begin{array}{l}\text { Mean value } \\
(\mathrm{mm})\end{array}$ & $\begin{array}{l}\text { Minimum } \\
(\mathrm{mm})\end{array}$ & $\begin{array}{l}\text { Maximum } \\
(\mathrm{mm})\end{array}$ & $\begin{array}{l}2 \mathrm{SD} \\
(\mathrm{mm})\end{array}$ \\
\hline $\begin{array}{l}\text { Biceps tendon (long head): transverse } \\
\text { diameter }\end{array}$ & Biceps tendon: transverse & Distal end of rotator cuff & 5.0 & 2.1 & 8.8 & 2.1 \\
\hline $\begin{array}{l}\text { Biceps tendon (long head): sagittal } \\
\text { diameter }\end{array}$ & Biceps tendon: longitudinal & Distal end of rotator cuff & 2.6 & 1.5 & 6.0 & 1.4 \\
\hline $\begin{array}{l}\text { Biceps tendon (long head): hypoechoic } \\
\text { rim }\end{array}$ & Biceps tendon: longitudinal & $\begin{array}{l}\text { Localisation of maximum } \\
\text { diameter }\end{array}$ & 1.4 & 0.5 & 5.3 & 1.4 \\
\hline Subscapularis tendon: sagittal diameter & $\begin{array}{l}\text { Shoulder: anterior transverse, max. } \\
\text { external rotation }\end{array}$ & $\begin{array}{l}2 \mathrm{~cm} \text { medial of biceps } \\
\text { tendon }\end{array}$ & 4.2 & 2.4 & 6.5 & 1.6 \\
\hline Supraspinatus tendon: sagittal diameter & $\begin{array}{l}\text { Shoulder: anterior transverse, } 60^{\circ} \\
\text { internal rotation }\end{array}$ & $\begin{array}{l}2 \mathrm{~cm} \text { lateral of biceps } \\
\text { tendon }\end{array}$ & 4.6 & 1.6 & 8.0 & 1.9 \\
\hline $\begin{array}{l}\text { Humeral head: cartilage below the } \\
\text { supraspinatus tendon }\end{array}$ & $\begin{array}{l}\text { Shoulder: anterior transverse, } 60^{\circ} \\
\text { internal rotation }\end{array}$ & $\begin{array}{l}2 \mathrm{~cm} \text { lateral of biceps } \\
\text { tendon }\end{array}$ & 0.8 & 0.2 & 3.0 & 0.6 \\
\hline Infraspinatus tendon: sagittal diameter & $\begin{array}{l}\text { Shoulder: posterior transverse, } 60^{\circ} \\
\text { internal rotation }\end{array}$ & Maximum posterior point & 3.8 & 1.9 & 6.9 & 1.8 \\
\hline $\begin{array}{l}\text { Distance between humeral head and } \\
\text { acromion }\end{array}$ & $\begin{array}{l}\text { Shoulder: lateral longitudinal, } 60^{\circ} \\
\text { internal rotation }\end{array}$ & $\begin{array}{l}\text { Strictly lateral (cranial } \\
\text { side of acromion) }\end{array}$ & 10.9 & 5.9 & 19.6 & 4.2 \\
\hline Humeral head: erosion longitudinal & $\begin{array}{l}\text { Shoulder: anterior, lateral or } \\
\text { posterior longitudinal }\end{array}$ & On site of greatest erosion & 3.9 & 1.1 & 11.4 & 4.8 \\
\hline Humeral head: erosion transverse & $\begin{array}{l}\text { Shoulder: anterior, lateral or } \\
\text { posterior transverse }\end{array}$ & On site of greatest erosion & 3.7 & 1.0 & 9.4 & 3.7 \\
\hline Humeral head: erosion sagittal & $\begin{array}{l}\text { Shoulder: anterior, lateral or } \\
\text { posterior transverse }\end{array}$ & On site of greatest erosion & 2.3 & 0.8 & 5.1 & 1.8 \\
\hline Subdeltoid bursa: sagittal & Shoulder: lateral longitudinal & Maximum diameter & 0.5 & 0.1 & 2.7 & 0.6 \\
\hline Axillary recess: sagittal & Axillary recess: longitudinal & $\begin{array}{l}\text { Middle of concavity of } \\
\text { humeral neck }\end{array}$ & 2.2 & 0.4 & 6.2 & 1.6 \\
\hline $\begin{array}{l}\text { Acromioclavicular joint, bone-capsule } \\
\text { distance: medial }\end{array}$ & Acromioclavicular joint: transverse & Lateral end of clavicula & 1.7 & 0.4 & 4.3 & 1.4 \\
\hline $\begin{array}{l}\text { Acromioclavicular joint bone-capsule } \\
\text { distance: lateral }\end{array}$ & Acromioclavicular joint: transverse & Medial end of acromion & 2.5 & 0.4 & 5.0 & 1.7 \\
\hline Acromioclavicular joint space & Acromioclavicular joint: transverse & $\begin{array}{l}\text { Cranial aspect: clavicula/ } \\
\text { acromion }\end{array}$ & 5.2 & 1.8 & 11.3 & 3.3 \\
\hline $\begin{array}{l}\text { Sternoclavicular joint, bone-capsule } \\
\text { distance: medial }\end{array}$ & Sternoclavicular joint: oblique & $\begin{array}{l}\text { Cranial lateral end of } \\
\text { sternum }\end{array}$ & 0.9 & 0.1 & 3.8 & 1.3 \\
\hline $\begin{array}{l}\text { Sternoclavicular joint bone-capsule } \\
\text { distance: lateral }\end{array}$ & Sternoclavicular joint: oblique & Medial end of clavicula & 1.5 & 0.1 & 4.3 & 1.6 \\
\hline Sternoclavicular joint, joint space & Sternoclavicular joint: oblique & $\begin{array}{l}\text { Anterior aspect: sternum/ } \\
\text { clavicula }\end{array}$ & 8.2 & 1.5 & 15.6 & 5.3 \\
\hline
\end{tabular}

at the volar proximal aspects of the MCP and proximal interphalangeal (PIP) II joints, respectively. We found no erosions at the MCP II and PIP II joints when examining the joints circumferentially with a longitudinal scan. We detected a hypoechoic rim around 198 (97\%) of the extensor carpi ulnaris tendons, and around $199(97 \%)$ of the index finger flexor tendons, with $2 \mathrm{SD}$ including $\leqslant 2.2 \mathrm{~mm}$ and $\leqslant 1.7 \mathrm{~mm}$, respectively.

\section{Hips}

The $2 \mathrm{SD}$ of the capsular distension in the anterior recess include distances $\leqslant 8.0 \mathrm{~mm}$. We found a hypoechoic rim in $176(86 \%)$ of the trochanteric bursae.

\section{Knees}

We detected fluid in 158 (77\%) of the suprapatellar recessi, in $96(47 \%)$ of the suprapatellar lateral recessi, in $12(6 \%)$ of the deep infrapatellar bursae, and in $32(16 \%)$ of the popliteal bursae. The mean thickness of the intercondylar cartilage was $2.7 \mathrm{~mm}(1.5-4.1 ; 2 \mathrm{SD}, 1.3)$ in women and $3.5 \mathrm{~mm}(2.0-4.9$; 2 SD 1.4) in men. The sagittal diameter of the patellar tendon was $2.9 \mathrm{~mm}(1.6-4.3 ; 2 \mathrm{SD}, 1.0 \mathrm{~mm})$ in women and $3.5 \mathrm{~mm}$ $(1.2-5.5 ; 2$ SD, 1.6) in men.

\section{Ankles, feet, and toes}

The 2 SD of the capsular distension include distances $\leqslant 2.1 \mathrm{~mm}$ in the anterior region and $\leqslant 2.3 \mathrm{~mm}$ in the posterior region of the ankle joint, $\leqslant 2.6 \mathrm{~mm}$ at the talonavicular joint, $\leqslant 3.5 \mathrm{~mm}$ in the first metatarsophalangeal joint, and $\leqslant 3.1 \mathrm{~mm}$ in the second metatarsophalangeal joint. We found a hypoechoic rim around the following: 138 $(68 \%)$ of the peroneus longus tendons, $156(76 \%)$ of the peroneus brevis tendons, $93(46 \%)$ of the tibialis anterior tendons, 91 (45\%) of the tibialis posterior tendons, and 26 (13\%) of the Achilles tendons. The mean sagittal diameter of the Achilles tendon was $4.1 \mathrm{~mm}(2.6-6.7 ; 2 \mathrm{SD}, 1.4)$ in women and $4.6 \mathrm{~mm}(3.0-6.4 ; 2 \mathrm{SD}, 1.6)$ in men. The mean transverse diameter was $13.3 \mathrm{~mm}(8.2-17.3 ; 2 \mathrm{SD}, 3.4)$ in women and $15.4 \mathrm{~mm}(9.4-20.6 ; 2 \mathrm{SD}, 3.9)$ in men. We detected fluid in $49(24 \%)$ retrocalcaneal bursae. The 2 SD of the sagittal diameter of the plantar fascia include distances $\leqslant 4.7 \mathrm{~mm}$.

\section{Correlation with age, sex, body weight, and height, sport activities, and hand dominance}

Owing to the large number of correlations investigated, several values reached significant levels $(p<0.05)$ as a result of an $\alpha$ fault. Only six of the correlations for differences between the sexes mentioned above were apparently relevant. There was no statistical significance adjusted to Holm between the dominant and non-dominant side, age $<40$ years and age $\geqslant 40$ years, and low and high working activity.

\section{Objectivity and reliability}

The rate of agreement between the two investigators (objectivity) was $0.96(0.78-0.99)$. It was $0.96(0.78-0.99)$ for tendon diameters; 0.95 (0.83-0.99) for capsular distensions at the joints; $0.97(0.94-0.99)$ for hypoechoic rims around tendons; $0.95(0.87-0.99)$ for joint spaces, erosions, and cartilage thickness; and $0.97(0.94-0.99)$ for bursae and 
Table 3 Anatomical structure with ultrasound plane; localisation of measurement; mean, maximum, and minimum value; and two standard deviations at the elbows, wrists, hands, and fingers

\begin{tabular}{|c|c|c|c|c|c|c|}
\hline Anatomical structure & Ultrasound plane & Exact localisation & $\begin{array}{l}\text { Mean value } \\
(\mathrm{mm})\end{array}$ & $\begin{array}{l}\text { Minimum } \\
(\mathrm{mm})\end{array}$ & $\begin{array}{l}\text { Maximum } \\
(\mathrm{mm})\end{array}$ & $\begin{array}{l}2 \mathrm{SD} \\
(\mathrm{mm})\end{array}$ \\
\hline $\begin{array}{l}\text { Elbow: coronoid fossa, bone-capsule } \\
\text { distance }\end{array}$ & Elbow: anterior longitudinal & Bottom of coronoid fossa & 1.8 & 0.3 & 5.9 & 1.9 \\
\hline $\begin{array}{l}\text { Elbow: anterior ulnar, bone-capsule } \\
\text { distance }\end{array}$ & Elbow: anterior transverse & Most anterior ulnar point & 1.0 & 0.6 & 2.5 & 0.5 \\
\hline $\begin{array}{l}\text { Elbow: olecranon fossa, bone-capsule } \\
\text { distance }\end{array}$ & $\begin{array}{l}\text { Elbow: posterior longitudinal, } \\
\text { maximum flexion }\end{array}$ & $\begin{array}{l}\text { Bottom of olecranon fossa, } \\
\text { midline }\end{array}$ & 1.9 & 0.2 & 4.8 & 2.0 \\
\hline $\begin{array}{l}\text { Wrist: radiocarpal joint, volar bone- } \\
\text { capsule distance }\end{array}$ & Wrist: volar longitudinal radial & $\begin{array}{l}\text { Scaphoid bone } 1 \mathrm{~cm} \text { distal } \\
\text { of joint }\end{array}$ & 1.7 & 0.2 & 4.9 & 1.7 \\
\hline $\begin{array}{l}\text { Wrist: radiocarpal joint, dorsal bone- } \\
\text { capsule distance }\end{array}$ & Wrist: dorsal longitudinal radial & $\begin{array}{l}\text { Scaphoid bone } 1 \mathrm{~cm} \text { distal } \\
\text { of joint }\end{array}$ & 1.5 & 0.2 & 3.7 & 1.4 \\
\hline $\begin{array}{l}\text { Wrist: head of the ulna, volar bone- } \\
\text { capsule distance }\end{array}$ & Wrist: volar longitudinal ulnar & Ulna $1 \mathrm{~cm}$ proximal of joint & 1.1 & 0.2 & 3.4 & 1.0 \\
\hline $\begin{array}{l}\text { Wrist: head of the ulna, dorsal bone- } \\
\text { capsule distance }\end{array}$ & Wrist: dorsal longitudinal ulnar & $\begin{array}{l}\text { Maximum dorsal point of } \\
\text { ulna }\end{array}$ & 0.8 & 0.1 & 2.5 & 0.8 \\
\hline $\begin{array}{l}\text { Wrist: extensor carpi ulnaris tendon } \\
\text { transverse diameter }\end{array}$ & Wrist: dorsal transverse ulnar & $\begin{array}{l}\text { Directly distal of the head } \\
\text { of the ulna }\end{array}$ & 5.4 & 1.8 & 9,0 & 2.6 \\
\hline $\begin{array}{l}\text { Wrist: extensor carpi ulnaris tendon } \\
\text { sagittal diameter }\end{array}$ & Wrist: dorsal transverse ulnar & $\begin{array}{l}\text { Directly distal of the head } \\
\text { of the ulna }\end{array}$ & 2.7 & 1.2 & 7.0 & 2.1 \\
\hline $\begin{array}{l}\text { Wrist: extensor carpi ulnaris tendon } \\
\text { hypoechoic rim }\end{array}$ & Wrist: dorsal transverse ulnar & $\begin{array}{l}\text { Directly distal of the head } \\
\text { of the ulna }\end{array}$ & 1.2 & 0.2 & 3.8 & 1.0 \\
\hline $\begin{array}{l}\text { Hand: index finger flexor tendons } \\
\text { transverse diameter }\end{array}$ & Hand: volar transverse & $\begin{array}{l}1 \mathrm{~cm} \text { proximal of MCP II } \\
\text { joint }\end{array}$ & 6.4 & 2.4 & 13.6 & 2.7 \\
\hline $\begin{array}{l}\text { Hand: index finger flexor tendons } \\
\text { sagittal diameter }\end{array}$ & Hand: volar transverse & $\begin{array}{l}1 \mathrm{~cm} \text { proximal of } \mathrm{MCP} \| \\
\text { joint }\end{array}$ & 3.6 & 0.6 & 9.3 & 2.2 \\
\hline $\begin{array}{l}\text { Hand: index finger flexor tendons } \\
\text { hypoechoic rim }\end{array}$ & Hand: volar transverse & $\begin{array}{l}1 \mathrm{~cm} \text { proximal of MCP } \| \\
\text { joint }\end{array}$ & 0.9 & 0.2 & 3.9 & 0.8 \\
\hline $\begin{array}{l}\text { MCP II joint: maximum bone-capsule } \\
\text { distance }\end{array}$ & MCP II: joint volar longitudinal & Region proximal of MCP II & 0.9 & 0.3 & 2.7 & 1.0 \\
\hline $\begin{array}{l}\text { PIP II joint: maximum bone-capsule } \\
\text { distance }\end{array}$ & PIP II: joint volar longitudinal & Region proximal of PIP II & 0.8 & 0.1 & 2.3 & 0.8 \\
\hline
\end{tabular}

Table 4 Anatomical structure with ultrasound plane; localisation of measurement; mean, maximum, and minimum value; and two standard deviations at the hips and knees

\begin{tabular}{|c|c|c|c|c|c|c|}
\hline Anatomical structure & Ultrasound plane & Exact localisation & $\begin{array}{l}\text { Mean value } \\
(\mathrm{mm})\end{array}$ & $\begin{array}{l}\text { Minimum } \\
(\mathrm{mm})\end{array}$ & $\begin{array}{l}\text { Maximum } \\
(\mathrm{mm})\end{array}$ & $\begin{array}{l}2 \mathrm{SD} \\
(\mathrm{mm})\end{array}$ \\
\hline Hip joint: bone-capsule distance & $\begin{array}{l}\text { Hip joint: anterior longitudinal } \\
\text { parallel to femoral neck }\end{array}$ & $\begin{array}{l}\text { Middle of anterior concavity } \\
\text { of femoral neck }\end{array}$ & 5.2 & 1.1 & 9.0 & 2.8 \\
\hline $\begin{array}{l}\text { Trochanteric bursa: bone-capsule } \\
\text { distance }\end{array}$ & Major trochanter: transverse & $\begin{array}{l}\text { Most lateral aspect of major } \\
\text { trochanter }\end{array}$ & 1.1 & 0.2 & 5.0 & 1.1 \\
\hline $\begin{array}{l}\text { Knee: suprapatellar recess midline, } \\
\text { longitudinal diameter }\end{array}$ & $\begin{array}{l}\text { Knee: anterior suprapatellar mid-line } \\
\text { longitudinal }\end{array}$ & Midline aspect suprapatellar & 22.2 & 4.4 & 51.2 & 20.1 \\
\hline $\begin{array}{l}\text { Knee: suprapatellar recess midline, } \\
\text { transverse diameter }\end{array}$ & $\begin{array}{l}\text { Knee: anterior suprapatellar mid-line } \\
\text { transverse }\end{array}$ & $\begin{array}{l}\text { At maximum transverse } \\
\text { diameter }\end{array}$ & 13.8 & 1.3 & 37.6 & 14.4 \\
\hline $\begin{array}{l}\text { Knee: suprapatellar recess midline, } \\
\text { sagittal diameter }\end{array}$ & $\begin{array}{l}\text { Knee: anterior suprapatellar mid-line } \\
\text { longitudinal }\end{array}$ & $\begin{array}{l}\text { At maximum longitudinal } \\
\text { diameter }\end{array}$ & 2.4 & 0.5 & 7.1 & 2.4 \\
\hline $\begin{array}{l}\text { Knee: suprapatellar recess, lateral } \\
\text { longitudinal diameter }\end{array}$ & $\begin{array}{l}\text { Knee: anterior suprapatellar lateral } \\
\text { longitudinal }\end{array}$ & At lateral femoral epicondylus & 22.8 & 4.4 & 52.8 & 23.0 \\
\hline $\begin{array}{l}\text { Knee: suprapatellar recess, lateral } \\
\text { transverse diameter }\end{array}$ & $\begin{array}{l}\text { Knee: anterior suprapatellar lateral } \\
\text { transverse }\end{array}$ & At lateral femoral epicondylus & 10.8 & 1.7 & 37.7 & 13.5 \\
\hline $\begin{array}{l}\text { Knee: suprapatellar recess, lateral } \\
\text { sagittal diameter }\end{array}$ & $\begin{array}{l}\text { Knee: anterior suprapatellar lateral } \\
\text { longitudinal }\end{array}$ & At lateral femoral epicondylus & 2.4 & 0.6 & 6.8 & 2.5 \\
\hline Knee: cartilage sagittal diameter & $\begin{array}{l}\text { Knee: anterior suprapatellar } \\
\text { transverse, } 90^{\circ} \text { flexion }\end{array}$ & Directly proximal of patella & 3.1 & 1.5 & 4.9 & 1.4 \\
\hline $\begin{array}{l}\text { Knee: patellar tendon sagittal } \\
\text { diameter }\end{array}$ & $\begin{array}{l}\text { Knee: anterior infrapatellar } \\
\text { longitudinal }\end{array}$ & $\begin{array}{l}2 \mathrm{~cm} \text { distal of distal end of } \\
\text { patella }\end{array}$ & 3.2 & 1.2 & 5.5 & 1.3 \\
\hline $\begin{array}{l}\text { Knee: deep infrapatellar bursa } \\
\text { longitudinal diameter }\end{array}$ & $\begin{array}{l}\text { Knee: anterior infrapatellar } \\
\text { longitudinal }\end{array}$ & Maximum longitudinal diameter & 6.1 & 2.4 & 10.4 & 4.4 \\
\hline $\begin{array}{l}\text { Knee: deep infrapatellar bursa } \\
\text { transverse diameter }\end{array}$ & $\begin{array}{l}\text { Knee: anterior infrapatellar } \\
\text { transverse }\end{array}$ & Maximum transverse diameter & 6.2 & 2.5 & 14.5 & 6.2 \\
\hline $\begin{array}{l}\text { Knee: deep infrapatellar bursa } \\
\text { sagittal diameter }\end{array}$ & $\begin{array}{l}\text { Knee: anterior infrapatellar } \\
\text { longitudinal }\end{array}$ & Maximum sagittal diameter & 2.7 & 0.7 & 12.0 & 6.1 \\
\hline $\begin{array}{l}\text { Knee: popliteal bursa longitudinal } \\
\text { diameter }\end{array}$ & Knee: posterior longitudinal & Maximum longitudinal diameter & 22.2 & 5.6 & 52.7 & 21.0 \\
\hline $\begin{array}{l}\text { Knee: popliteal bursa transverse } \\
\text { diameter }\end{array}$ & Knee: posterior transverse & Maximum transverse diameter & 10.1 & 1.6 & 29.5 & 14.6 \\
\hline $\begin{array}{l}\text { Knee: popliteal bursa sagittal } \\
\text { diameter }\end{array}$ & Knee: posterior transverse & Maximum sagittal diameter & 5.2 & 1.2 & 23.6 & 8.6 \\
\hline
\end{tabular}


Table 5 Anatomical structure with ultrasound plane; localisation of measurement; mean, maximum, and minimum value; and two standard deviations at the ankles, feet, and toes

\begin{tabular}{|c|c|c|c|c|c|c|}
\hline Anatomical structure & Ultrasound plane & Exact localisation & $\begin{array}{l}\text { Mean value } \\
(\mathrm{mm})\end{array}$ & $\begin{array}{l}\text { Minimum } \\
(\mathrm{mm})\end{array}$ & $\begin{array}{l}\text { Maximum } \\
(\mathrm{mm})\end{array}$ & $2 \mathrm{SD}(\mathrm{mm})$ \\
\hline $\begin{array}{l}\text { Tibiotalar joint: anterior bone-capsule } \\
\text { distance }\end{array}$ & $\begin{array}{l}\text { Ankle: anterior longitudinal } \\
\text { midline }\end{array}$ & $1 \mathrm{~cm}$ distal of tibiotalar joint & 1.1 & 0.4 & 3.8 & 1.0 \\
\hline $\begin{array}{l}\text { Tibiotalar joint: posterior bone-capsule } \\
\text { distance }\end{array}$ & $\begin{array}{l}\text { Ankle: posterior longitudinal } \\
\text { midline }\end{array}$ & Directly distal of tibiotalar joint & 1.2 & 0.3 & 3.7 & 1.1 \\
\hline $\begin{array}{l}\text { Talonavicular joint: anterior bone-capsule } \\
\text { distance }\end{array}$ & $\begin{array}{l}\text { Ankle: anterior longitudinal } \\
\text { midline }\end{array}$ & Maximum diameter & 1.4 & 0.4 & 4.2 & 1.2 \\
\hline Tibialis anterior tendon: transverse diameter & Ankle: anterior transverse & At tibiotalar joint & 8.2 & 5.0 & 14.2 & 3.5 \\
\hline Tibialis anterior tendon: sagittal diameter & Ankle: anterior transverse & At tibiotalar joint & 2.5 & 1.1 & 4.5 & 1.3 \\
\hline Tibialis anterior tendon: hypoechoic rim & Ankle: anterior transverse & At tibiotalar joint & 0.8 & 0.1 & 2.8 & 0.9 \\
\hline $\begin{array}{l}\text { Tibialis posterior tendon: transverse } \\
\text { diameter }\end{array}$ & Ankle: medial transverse & $\begin{array}{l}\text { Directly below level of medial } \\
\text { malleolus }\end{array}$ & 8.4 & 3.1 & 14.1 & 4.2 \\
\hline Tibialis posterior tendon: sagittal diameter & Ankle: medial transverse & $\begin{array}{l}\text { Directly below level of medial } \\
\text { malleolus }\end{array}$ & 2.8 & 1.3 & 6.0 & 1.8 \\
\hline Tibialis posterior tendon: hypoechoic rim & Ankle: medial transverse & $\begin{array}{l}\text { Directly below level of medial } \\
\text { malleolus }\end{array}$ & 1.2 & 0.2 & 3.8 & 1.6 \\
\hline $\begin{array}{l}\text { Peroneus longus tendon: transverse } \\
\text { diameter }\end{array}$ & Ankle: lateral transverse & $\begin{array}{l}\text { Directly below level of lateral } \\
\text { malleolus }\end{array}$ & 6.0 & 2.5 & 12.3 & 3.7 \\
\hline Peroneus longus tendon: sagittal diameter & Ankle: lateral transverse & $\begin{array}{l}\text { Directly below level of lateral } \\
\text { malleolus }\end{array}$ & 3.0 & 0.8 & 6.1 & 1.6 \\
\hline Peroneus longus tendon: hypoechoic rim & Ankle: lateral transverse & $\begin{array}{l}\text { Directly below level of lateral } \\
\text { malleolus }\end{array}$ & 1.1 & 0.2 & 4.1 & 1.2 \\
\hline $\begin{array}{l}\text { Peroneus brevis tendon: transverse } \\
\text { diameter }\end{array}$ & Ankle: lateral transverse & $\begin{array}{l}\text { Directly below level of lateral } \\
\text { malleolus }\end{array}$ & 4.3 & 1.8 & 13.0 & 3.0 \\
\hline Peroneus brevis tendon: sagittal diameter & Ankle: lateral transverse & $\begin{array}{l}\text { Directly below level of lateral } \\
\text { malleolus }\end{array}$ & 2.5 & 0.5 & 4.2 & 1.3 \\
\hline Peroneus brevis tendon: hypoechoic rim & Ankle: lateral transverse & $\begin{array}{l}\text { Directly below level of lateral } \\
\text { malleolus }\end{array}$ & 0.9 & 0.2 & 2.2 & 0.8 \\
\hline Achilles tendon: transverse diameter & Ankle: posterior transverse & $2 \mathrm{~cm}$ proximal of calcaneus & 14.3 & 8.2 & 20.6 & 4.1 \\
\hline Achilles tendon: sagittal diameter & Ankle: posterior longitudinal & $2 \mathrm{~cm}$ proximal of calcaneus & 4.3 & 2.6 & 6.7 & 1.6 \\
\hline Achilles tendon: hypoechoic rim & Ankle: posterior transverse & $2 \mathrm{~cm}$ proximal of calcaneus & 1.0 & 0.2 & 4.4 & 2.3 \\
\hline Retrocalcaneal bursa: longitudinal diameter & Ankle: posterior longitudinal & Maximum longitudinal diameter & 5.5 & 1.1 & 13.9 & 5.0 \\
\hline Retrocalcaneal bursa: transverse diameter & Ankle: posterior transverse & Maximum transverse diameter & 5.5 & 0.9 & 13.4 & 5.4 \\
\hline Retrocalcaneal bursa: sagittal diameter & Ankle: posterior longitudinal & Maximum sagittal diameter & 1.3 & 0.3 & 3.4 & 1.4 \\
\hline Plantar fascia: sagittal diameter & Plantar: longitudinal & At distal plantar end of calcaneus & 3.4 & 1.8 & 5.3 & 1.3 \\
\hline $\begin{array}{l}\text { MTP I joint: maximum bone-capsule } \\
\text { distance }\end{array}$ & $\begin{array}{l}\text { MTP I joint: anterior } \\
\text { longitudinal }\end{array}$ & Region proximal of MTP I & 1.7 & 0.2 & 4.8 & 1.8 \\
\hline $\begin{array}{l}\text { MTP II joint: maximum bone-capsule } \\
\text { distance }\end{array}$ & $\begin{array}{l}\text { MTP II joint: anterior } \\
\text { longitudinal }\end{array}$ & Region proximal of MTP \| & 1.6 & 0.4 & 3.9 & 1.5 \\
\hline
\end{tabular}

recesses. It was $0.96(0.87-0.99)$ for shoulders, $0.92(0.83-$ $0.99)$ for elbows, $0.96(0.89-0.99)$ for hands and fingers, 0.96 (0.94-0.99) for hips, $0.98(0.98-0.99)$ for knees, and 0.96 (0.78-0.99) for feet and toes.

The rate of agreement between follow up investigations of the same test subject (reliability) was $0.83(0.52-0.99)$. It was $0.87(0.74-0.99)$ for tendon diameters; $0.84(0.72-0.97)$ for capsular distensions at the joints; $0.86(0.78-0.96)$ for hypoechoic rims around tendons; $0.84(0.70-0.99)$ for joint spaces, erosions, and cartilage thickness; and $0.70(0.52-$ $0.85)$ for bursae and recesses. It was $0.86(0.70-0.97)$ for shoulders, $0.64(0.52-0.78)$ for elbows, $0.84(0.72-0.96)$ for hands and fingers, $0.82(0.70-0.93)$ for hips, $0.72(0.57-0.99)$ for knees, and $0.86(0.78-0.97)$ for feet and toes.

When the investigations were compared using different ultrasound equipment with less resolution the reliability was lower: $0.60(0.04-0.99)$. Distances generated with the second piece of equipment were greater and lower in half of the measurements, respectively. Thus we did not find a systematic fault with older equipment, but measurement was less exact.

\section{DISCUSSION}

Ultrasonography has been extensively considered as a primarily qualitative diagnostic tool. ${ }^{14}$ Qualitative measures of course remain important-for example, echogenicity, appearance of neighbouring structures, as well as comparison with the contralateral side and with clinical findings. Only a few small studies have published definitions of standard reference values in selected anatomical regions. The percentage of detected structures that contain a small amount of fluid is consequently much lower in most publications than in our study. The space between articular surfaces is relatively small in most joints. Most articular fluid is usually distributed throughout joint recesses, where it can be assessed by ultrasonography. A small amount of fluid is also usually detectable in tendon sheaths, where it appears as a thin hypoechoic rim. Advanced high resolution scanners are, in contrast with older equipment, capable of detecting fine details in anatomical structures that demonstrate only very slight deviation from the normal. ${ }^{15}$

In asymptomatic shoulders, Arslan et al detected biceps tendon fluid in only $8 \%$ of cases, and subdeltoid bursal fluid in only 3\%. ${ }^{16}$ A magnetic resonance imaging (MRI) study found fluid in $20 \%$ of subdeltoid bursae. ${ }^{17}$ Cartilage thickness at the humeral head was slightly higher in cadavers $(1.2 \mathrm{~mm})$ than in our subjects $(0.8 \mathrm{~mm}) \cdot{ }^{18}$ Wallny et al found a similar mean sagittal diameter of the biceps tendon ( $2.8 \mathrm{~mm} v 2.6 \mathrm{~mm}$ in our study), and a larger diameter of the supraspinatus tendon $(6.25 \mathrm{~mm})$ in 50 healthy volunteers. ${ }^{19}$ Koski described the axillar scan of the glenohumeral joint and arrived at similar diameters for capsular distension (2.4 mm $v 2.2 \mathrm{~mm}$ in our study). ${ }^{20}$ Alasaarela et al likewise obtained similar diameters for capsular distension $(2.2 \mathrm{~mm} \mathrm{v}$ $1.7 / 2.5 \mathrm{~mm}$ in our study) and for joint space $(4.1 \mathrm{~mm} v$ $5.2 \mathrm{~mm}$ in our study) for the acromioclavicular joint. ${ }^{21}$

In studies of elbows in healthy subjects, Koski described anterior capsular-distension distances up to $2 \mathrm{~mm} .{ }^{22}$ These 
findings are comparable to our data at the ulna (2 SD $\leqslant 1.5 \mathrm{~mm}$ ).

Hands, wrists, and fingers are frequently the subjects of investigation, particularly in rheumatology. To our knowledge, however, no studies have published measured distances in a healthy population. In concurrence with other studies, we detected no erosions at the MCP and PIP joints in healthy subjects. ${ }^{53}$ This finding is very important as ultrasonography is a new method used specifically to detect erosions in early rheumatoid arthritis that may not be seen by conventional radiography. In contrast, we found erosions of the humeral head in many healthy adults. Therefore it is not possible to draw any clinical conclusion if ultrasonography detects smaller erosions of the humeral head.

An MRI study of asymptomatic hip joints has described distances $\leqslant 5 \mathrm{~mm}$ between femur and joint capsule. ${ }^{24}$ In the recess anterior to the femoral neck, Koski et al suggested a larger upper limit of $7 \mathrm{~mm},{ }^{25}$ which is slightly lower than our findings ( $2 \mathrm{SD}$ includes diameters $\leqslant 8.0 \mathrm{~mm}$ ). Sada suggested an upper limit of $9 \mathrm{~mm} .{ }^{26}$ In knee studies, Mielke et al found a smaller diameter of the intercondylar cartilage (mean $2.4 \mathrm{~mm} v 3.1 \mathrm{~mm}$ ). This study did not detect popliteal cysts in a normal population owing to lower resolution with older equipment. ${ }^{27}$

MRI studies have described fluid in normal ankle joints and in tendon sheaths around the ankles. ${ }^{28}$ Nazarian et al disclosed fluid in the anterior recess of the ankle joint in 33\% of subjects examined. ${ }^{29}$ In addition to our findings and those of two other studies, ${ }^{28}{ }^{30}$ Nazarian could not detect fluid in the posterior recess. In contrast, their study found retrocalcaneal bursal fluid in $50 \%$ of subjects, compared with $24 \%$ in our findings. Their study also found tibialis posterior tendon sheath fluid in $77 \%$ of tendons. Another study revealed fluid in $85 \%$ of tendons ${ }^{31}$ - compared with $45 \%$ in our study. The smaller number of tendons with tendon sheath fluid in our study can be explained by the fact that we only used one fixed localisation to evaluate the tendon. Seybold and Hamel found fluid in the peroneal tendon sheaths in only $7 \%$ of subjects, compared with 68 and $76 \%$ in our study. ${ }^{31}$ The sagittal diameter of the Achilles tendon was $4.9 \mathrm{~mm}$ for women and $5.3 \mathrm{~mm}$ for men in a Spanish study, ${ }^{32}$ compared with $4.1 \mathrm{~mm}$ and $4.6 \mathrm{~mm}$, respectively, in our study.

Another small study from 1988 described even larger diameters-namely, $6.2 \mathrm{~mm}(4-9) .^{33}$ We have not encountered such large diameters in our studies. For the plantar fascia, our diameters $(3.4 \mathrm{~mm})$ are comparable to those described in other studies: $2.9 \mathrm{~mm},{ }^{34} 3.2 \mathrm{~mm},{ }^{35}$ and $3.3 \mathrm{~mm} .{ }^{36}$ Kamel and Kotob described only a mean diameter of $2.3 \mathrm{~mm}$ in 20 healthy Egyptian subjects. ${ }^{37}$ The diameters for capsular distension at the toes are comparable to those found in another study. The authors suggested that distances $>3 \mathrm{~mm}$ are pathological. ${ }^{38}$

In our study we always carried out measurements at the same defined location; distances may therefore be larger in other areas of anatomical structures. In addition, we investigated only 20-60 year old subjects. Distances in younger subjects are different before epiphysial closure and because of a thicker layer of cartilage. In older people, distances may differ as a result of an increasing prevalence of osteoarthritis and a reduction of cartilage thickness. Our test subjects, furthermore, had obtained a higher education level and practised more sedentary professions than the general population. Nevertheless, we found no correlation of our data with the sports activities of our subjects. Selected distances are different for athletes. ${ }^{39}$ Body weight and height of our subjects are comparable to data for the American population aged $\geqslant 20$ years, according to findings for population prevalence estimates published by the Third National Health and Nutrition Examination Survey. ${ }^{40}$ The weight of our subjects was $62 \mathrm{~kg}$ for women and $80 \mathrm{~kg}$ for men, compared with $66 \mathrm{~kg}$ and $80 \mathrm{~kg}$ in the American population. Height was $166 \mathrm{~cm}$ for women and $179 \mathrm{~cm}$ for men compared with $162 \mathrm{~cm}$ and $176 \mathrm{~cm}$ in the American population.

Although this is the first study which systematically measures distances of healthy adults in all anatomical regions that are important for musculoskeletal ultrasound, further structures remain to be evaluated. We chose those distances that appeared to be most relevant for clinical practice according to our experience. We did not investigate the median and the ulnar nerves because sufficient data have already been published by other authors. ${ }^{41-43}$ We cannot exclude the possibility that the values for the MCP, PIP, and metatarsophalangeal joints that we did not investigate may slightly differ from those of the joints that we chose for this study.

Our study aims at defining a normal population. In accordance with most other studies that have described normal values of musculoskeletal ultrasonography we determined mean values and standard deviations. Analysis of receiver operating characteristic curves can be performed to define more exact standard values that distinguish a well defined disease from a normal population in a selected anatomical structure. This has been investigated in the comparison of Achilles tendon diameters of patients with familial hypercholesterolaemia with diameters of subjects with other lipid profiles. ${ }^{44}$

Studies that investigated distances with MRI arrived at similar results to those obtained with ultrasonography. ${ }^{17} 182428{ }^{35}$ Comparison with necropsy studies is difficult because of reduced synovial and interstitial fluid. ${ }^{27}$

In conclusion, hypoechoic rims within joints and around tendons, fluid in bursae, and erosions at the humeral head are common findings in healthy subjects. It is important to define standard reference values for musculoskeletal ultrasonography to prevent misinterpretation of normal fluid as articular or tendon abnormality.

\section{ACKNOWLEDGEMENTS}

The study was supported by a grant from the Association of the Cooperative Rheumatology Centres in the German Society for Rheumatology. The ultrasound equipment was provided for this study by Esaote SpA, Via Siffredi 58, I-16153 Genua, Italy.

\section{Authors' affiliations}

W A Schmidt, H Schmidt, E Gromnica-Ihle, Medical Centre for Rheumatology Berlin-Buch, Karower Strasse 11, 13125 Berlin, Germany B Schicke, Department of Biostatistics, HELIOS Klinikum Berlin, Klinikum Buch, Wiltbergstr 50, 13125 Berlin, Germany

\section{REFERENCES}

1 Showstack JA, Schroeder SA, Matsumoto MF. Changes in the use of medical technologies, 1972-1977: a study of 10 inpatient diagnoses. N Engl J Med 1982;306:706-12.

2 Manger B, Kalden JR. Joint and connective tissue ultrasonography-a rheumatologic bedside procedure? A German experience. Arthritis Rheum 1995;38:736-42.

3 Grassi W, Cervini C. Ultrasonography in rheumatology: an evolving technique. Ann Rheum Dis 1998;57:268-71.

4 Gibbon WW, Wakefield RJ. Ultrasound in inflammatory disease. Radiol Clin North Am 1999;37:633-51.

5 Schmidt WA. Value of sonography in diagnosis of rheumatoid arthritis. Lancet 2001;357:1056-7.

6 Karim Z, Wakefield RJ, Conaghan PG, Lawson CA, Goh E, Quinn MA, et al. The impact of ultrasonography on diagnosis and management of patients with musculoskeletal conditions. Arthritis Rheum 2001;44:2932-3.

7 Lund PJ, Nisbet JK, Valencia FG, Ruth JT. Current sonographic applications in orthopedics. AJR Am J Roentgenol 1996;166:889-95.

8 Jacobson JA, Lax MJ. Musculoskeletal sonography of the postoperative orthopedic patient. Semin Musculoskelet Radiol 2002;6:67-77.

9 Jacobson JA. Ultrasound in sports medicine. Radiol Clin North Am 2002;40:363-86. 
10 Healy JC. The value of ultrasound in sports medicine. Hosp Med 2002;63:593-7.

11 Katayose M, Magee DJ. The cross-sectional area of supraspinatus as measured by diagnostic ultrasound. J Bone Joint Surg Br 2001;83:565-8.

12 Feldman F. Musculoskeletal radiology: then and now. Radiology 2000;216:309-16.

13 Backhaus M, Burmester GR, Gerber T, Grassi W, Machold KP, Swen WA, et al. Guidelines for musculoskeletal ultrasound in rheumatology. Ann Rheum Dis 2001;60:641-9.

14 Grassi W, Filippucci E, Farina A, Cervini C. Sonographic imaging of tendons. Arthritis Rheum 2000;43:969-76.

15 Schmidt WA, Kraft HE, Vorpahl K, Völker L, Gromnica-Ihle EJ. Color duplex ultrasonography in the diagnosis of temporal arteritis. N Engl J Med 1997:337:1336-42.

16 Arslan G, Apaydin A, Kabaalioglu A, Sindel T, Luleci E. Sonographically detected subacromial/subdeltoid bursal effusion and biceps tendon sheath fluid: reliable signs of rotator cuff tear? J Clin Ultrasound 1999;27:335-9.

17 Neumann CH, Holt RG, Steinbach LS, Jahnke AH Jr, Petersen SA. MR imaging of the shoulder: appearance of the supraspinatus tendon in asymptomatic volunteers. AJR Am J Roentgenol 1992;158:1281-7.

18 Hodler J, Loredo RA, Longo C, Trudell D, Yu JS, Resnick D. Assessment of articular cartilage thickness of the humeral head: MR-anatomic correlation in cadavers. AJR Am J Roentgenol 1995;165:615-20.

19 Wallny T, Wagner UA, Prange S, Schmitt O, Reich H. Evaluation of chronic tears of the rotator cuff by ultrasound. A new index. J Bone Joint Surg $\mathrm{Br}$ 1999;81:675-8.

20 Koski JM. Axillar ultrasound of the glenohumeral joint. J Rheumatol 1989;19:664-7.

21 Alasaarela E, Tervonen O, Takalo R, Lahde S, Suramo I. Ultrasound evaluation of the acromioclavicular joint. J Rheumatol 1997;24:1959-63.

22 Koski JM. Ultrasonography of the elbow joint. Rheumatol Int 1990;10:91-4.

23 Wakefield RJ, Gibbon WW, Conaghan PG, O'Connor P, McGonagle D, Pease $C$, et al. The value of sonography in the detection of bone erosions in patients with rheumatoid arthritis: a comparison with conventional radiography. Arthritis Rheum 2000;43:2762-70.

24 Moss SG, Schweitzer ME, Jacobson JA, Brossmann J, Lombardi JV, Dellose SM, et al. Hip joint fluid: detection and distribution at MR imaging and US with cadaveric correlation. Radiology 1998;208:43-8.

25 Koski JM, Anttila PJ, Isomaki HA. Ultrasonography of the adult hip joint. Scand J Rheumatol 1989;18:113-17.

26 Sada PN, Rajan P, Jeyaseelan L, Washburn MC. Standards for ultrasonographic measurements of the hip joint in Indian adults. Skeletal Radiol 1994;23:111-12.

27 Mielke G, Brandrup-Lukanow A, Bandilla K, Berg D, Higer P, Loch EG. Ultrasound of the knee joint: normal values and changes in rheumatoid arthritis. Ultraschall Med 1990;11:40-3.
28 Schweitzer ME, van Leersum M, Ehrlich SS, Wapner K. Fluid in normal and abnormal ankle joints: amount and distribution as seen on MR images. AJR Am J Roentgenol 1994;162:111-14.

29 Nazarian LN, Rawool NM, Martin CE, Schweitzer ME. Synovial fluid in the hindfoot and ankle: detection of amount and distribution with US. Radiology 1995; 197:275-8

30 Friedrich JM, Schnarkowski P, Rubenacker S, Wallner B. Ultrasonography of capsular morphology in normal and traumatic ankle joints. J Clin Ultrasound 1993;21:179-87.

31 Seybold D, Hamel J. Standardized ultrasound diagnosis (13 MHz) of the tendon of the posterior tibial muscle-normal findings in probands with healthy feet. Z Orthop Ihre Grenzgeb 2000;138:269-77.

32 Civeira F, Castillo JJ, Calvo C, Ferrando J, de Pedro C, Martinez-Rodes P, et al. Achilles tendon size by high resolution sonography in healthy population. Relationship with lipid levels. Med Clin (Barc) 1998;1 1 1:41-4.

33 Mathieson JR, Connell DG, Cooperberg PL, Lloyd-Smith DR. Sonography of the Achilles tendon and adjacent bursae. AJR Am J Roentgenol 1988;151:127-31.

34 Cardinal E, Chhem RK, Beauregard CG, Aubin B, Pelletier M. Plantar fasciitis: sonographic evaluation. Radiology 1996;201:257-9.

35 Berkowitz JF, Kier R, Rudicel S. Plantar fasciitis: MR imaging. Radiology $1991 ; 179: 665-7$

36 Gibbon WW, Long G. Ultrasound of the plantar aponeurosis (fascia). Skeletal Radiol 1999;28:21-6.

37 Kamel M, Kotob H. High frequency ultrasonographic findings in plantar fasciitis and assessment of local steroid injection. J Rheumatol 2000;27:2139-41.

38 Koski JM. Ultrasonography of the metatarsophalangeal and talocrural joints. Clin Exp Rheumatol 1990;8:347-51.

39 Maffulli N, Regine R, Angelillo M, Capasso G, Filice S. Ultrasound diagnosis of Achilles tendon pathology in runners. Br J Sports Med 1987;21:158-62.

40 Kuczmarski RJ, Carroll MD, Flegal KM, Troiano RP. Varying body mass index cutoff points to describe overweight prevalence among U.S. adults: NHANES III (1988 to 1994), Obes Res 1997;5:542-8.

41 Lee D, van Holsbeeck MT, Janevski PK, Ganos DL, Ditmars DM, Darian VB. Diagnosis of carpal tunnel syndrome. Ultrasound versus electromyography. Radiol Clin North Am 1999;37:859-72.

42 Duncan I, Sullivan P, Lomas F. Sonography in the diagnosis of carpal tunnel syndrome. AJR Am J Roentgenol 1999;173:681-4.

43 Chiou HJ, Chou YH, Cheng SP, Hsu CC, Chan RC, Tiu CM, et al. Cubital tunnel syndrome: diagnosis by high-resolution ultrasonography. J Ultrasound Med 1998; 17:643-8

44 Descamps OS, Leysen X, van Leuven F, Heller FR. The use of Achilles tendon ultrasonography for the diagnosis of familial hypercholesterolemia. Atherosclerosis $2001 ; 157: 514-18$. 